\title{
Processes at Multi-pulse Laser Embossing of Submicron Surface Structures
}

\author{
Martin Ehrhardt, Pierre Lorenz, Frank Frost, Joachim Zajadacz, Klaus Zimmer \\ Leibniz Institute of Surface Modification, Permoserstr. 15, 04318 Leipzig, Germany \\ E-mail: martin.ehrhardt@iom-leipzig.de
}

\begin{abstract}
Three-dimensional micro- and nanostructuring of metal surfaces is requested due to the ongoing miniaturization and is therefore of great interest for microelectromechanical systems (MEMS), micro-optics, and precision machining. One promising fabrication method for low-cost, high-quality micro- and nanostructured metal surfaces is laser microembossing. Recent experimental results of laser embossing of submicron structures are presented to discuss the processes and characteristics of laser micro-embossing. Basically, laser embossing shows almost no modification caused by thermal effects of the laser pulses. Therefore, almost no burr, recast droplets, or near-surface material modifications have been found at the embossed structures. The elastoplastic deformation at incomplete submicron pattern embossing is probably the reason for the differences between the replicated pattern and the master structures. Unintended patterns like scratches and satellite patterns may occur in the case of multi-pulse laser embossing without carefully fixing the sample and master positions.

DOI: $10.2961 /$ jlmn.2014.03.0014
\end{abstract}

Keywords: laser embossing, submicron patterns, shock waves, plastic deformation

\section{Introduction}

The need for micro and nanostructured surfaces is rapidly grown within the last decades. This is the result of the steadily increasing number of applications making use of these patterned surfaces, for instance in the fields of optics, biomedicine, and micromechanics. Current methods for patterning of metal surfaces for microtechnology require photolithographic techniques and need either pattern transfer by etching or electroforming [1]. Laser patterning of metallic surfaces is feasible but suffers from particle generation that may induce defects in microsystems and from laser heating due to the absorbed laser energy that can cause melting of thin films or the surface of the workpiece [2]. Although ultrashort pulse lasers need less laser energy for material ablation material modification usually happens as can be concluded from changes of the threshold fluence with the laser pulse number [3]. Material modifications, contaminations by particles, debris or chips as well as material melting is disadvantageous for many applications in the field of micron technology. Hence, a flexible and lowcost manufacturing process which is suitable for $3 \mathrm{D}$ patterning of large metal surfaces for microtechnology is not available yet but is requested.

Replications by embossing technologies are often utilized for $3 \mathrm{D}$ patterning of polymers to enable a low-cost and high-throughput fabrication of patterned surfaces. For this purpose usually polymers softened by increased temperatures or liquid pre-polymers that can be cured are utilized for filling the small cavities of the moulds for patterning [4]. At these conditions moderate forces are sufficient to accomplish material flow.

For metals higher pressures are usually needed to get plastic forming. By means of temporary melting material surfaces the fabrication of submicrometer and nanostructures by laser-assisted direct imprinting (LADI) was demonstrat- ed recently [5]. However, the melting of the metal layer by laser pulses that is necessary for the replication may induce stress, cause material modifications, and may damage the moulds. Microembossing is a promising approach for replication of micron and submicron patterns into metal surfaces but requires master moulds, ductile workpiece metal, and high embossing pressures. Pulsed laser interaction with matter can cause mechanical processes such as stress formation, shockwave generation, and recoil pressures [2]. Already known applications of laser-generated shock waves are laser shock peening [6] that is used to increase the wear of metal surfaces and bending metallic microparts as several studies report [7-9]. Some physical processes such as shock wave generation by laser ablation, shock wave propagation, and interaction of shock waves with matter are similar in parts. Substantial differences to these known applications result from the utilization of a master surface to be replicated.

Up to now, only a few studies were published which deal with laser microembossing of 3D metallic structures at thin metal sheets $[10,11]$. In the papers of Cheng et al. the grain size reduction of thin metallic foils, the forming behaviour, and limitations due to the laser forming process were investigated [12-14]. Liu et al. investigate the application of micromould-based laser shock forming for MEMS applications $[10,15]$. The laser deep drawing process and the forming limits for metallic foils at micro deep drawing were studied by Vollertsen et al. [16, 17].

This study focuses on the laser embossing of submicron patterns into copper foils by means of multi-pulse irradiation and laser spot scanning. Such submicron-sized pattern is very interesting for optical applications.

\section{Experimental}

\subsection{Experimental set-up for laser microembossing}


In this study the standard experimental set-up for laser embossing is applied. This means that the master mould is below the sample for replication.
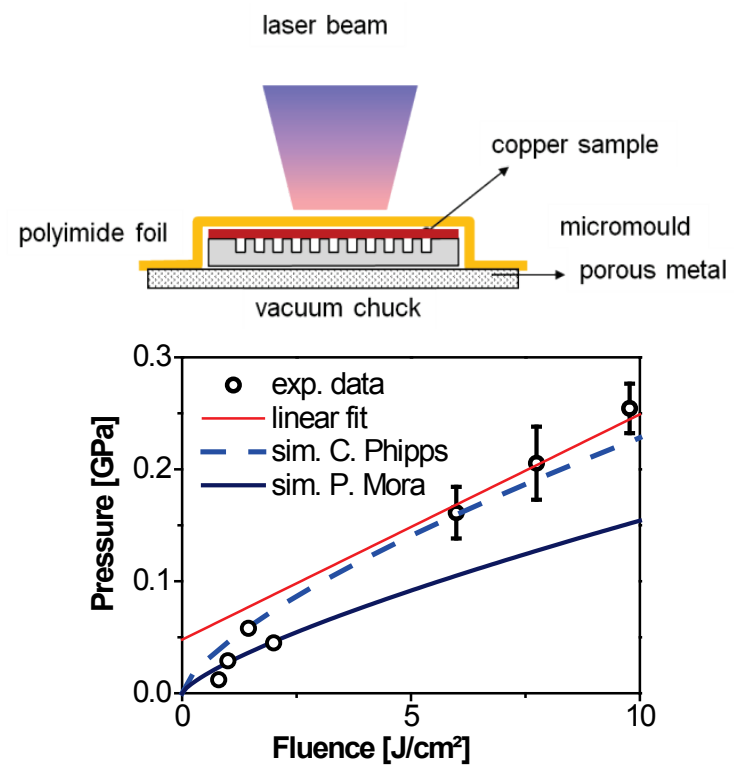

Figure 1: (top) Experimental set-up for laser embossing used in these experiments. The vacuum chuck fixes all parts of the experiment and enhances the pre-embossing contact between the surfaces of the sample and the mould. (bottom) Experimentally measured and simulated pressures generated by the laser ablation of the polyimide

In Figure 1 (top) the cross section of the experimental setup for laser microembossing is shown.

The mould was placed onto a vacuum chuck. The structured side of the mould (silicon, nickel shim) was face to face to the copper sample; a polyimide foil (PI) (thickness $\mathrm{d}=25 \mu \mathrm{m}$, UPILEX-25S) was used to seal the whole arrangement including the vacuum chuck. Because the polyimide foil covers up all pieces of the set-up the atmospheric pressure causes forces that compress the surfaces of the copper foil and the mould together. For replication a multipulse approach with scanning the laser beam with meanderlike patterns across the surface was utilized [18]. A $\mathrm{KrF}$ excimer laser with a pulse length of $\mathrm{tp}=25 \mathrm{~ns}$ and a wavelength of $\lambda=248 \mathrm{~nm}$, which is embedded in a laser workstation, was used for laser irradiation. The workstation provides a flat top beam profile at a laser spot of $A=100 \mu \mathrm{m} x$ $100 \mu \mathrm{m}$ that is scanned in a meandering pattern across the sample surface by means of a program-controlled $x-y-z$ stage. The repetition rate of the laser was fixed at a value of $\mathrm{f}=100 \mathrm{~Hz}$ and the laser fluence ranges from 3 to $5 \mathrm{~J} / \mathrm{cm}^{2}$. Due to the scanning approach the naturally limited size of the laser embossing area (due to the laser spot size) can be extended and shaped arbitrary so that additional technical applications can be addressed. The scanning velocity was adjusted in such a way to ensure a remaining polyimide foil thickness of approx. 1 to $2 \mu \mathrm{m}$ after completely scanning the sample. This remaining polyimide protects the copper sample from thermal impact of the laser beam because the optical penetration depth is less that $100 \mathrm{~nm}$ and the thermal conductivity is much less than that of copper. Moreover, the undamaged polyimide foil saves the sample from debris and contamination due to the laser ablation process and enables a clean processing that is necessary for micron and submicron patterning.

\subsection{Master and samples}

Within these experiments two different masters were utilized as moulds: a nickel foil featuring a submicron 2Dsine grating and silicon wafers processed with ultrashort pulse lasers for ripples formation. Submicron ripples in silicon surfaces have been used as masters, too. For preparation a linear polarized beam a $775 \mathrm{~nm}$ Ti:sapphire laser with a pulse length of 120 fs was defocussed and scanned across the sample. Fluences near the threshold and a pulse overlap of $>90 \%(\mathrm{~N}>10)$ were used to get laser-induced surface periodic structures (LIPSS) with a period similar to the wavelength $\lambda$ that are known to be low spatial frequency LIPSS (LSFL). The AFM image in Figure 2 shows the LIPSS with the typically wave-like patterns and some elevated features (shown as dark points in the inverted colour scale). These features are ejected material that is coalescent to the ripples pattern. The height of these patterns is significantly higher than the average peak of the ripples structures.

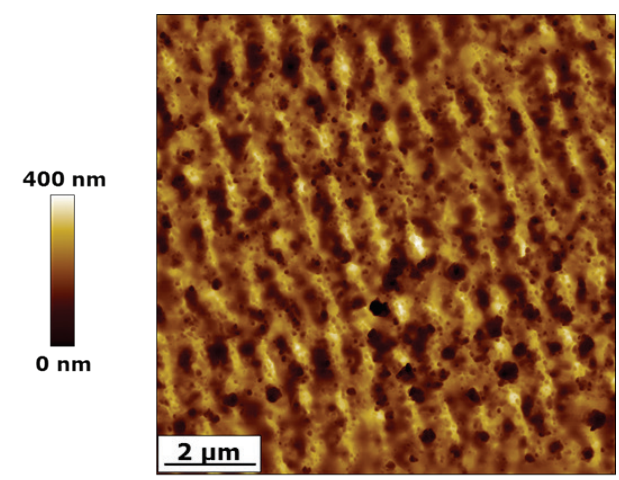

Figure 2: AFM image of the silicon master with LIPSS showing the colour-coded inverted height. In addition to the LSFL several droplet-like features are seen that can be assigned to be recast silicon. The height of these features is much larger than the P-V value of the LIPSS.

The topographies of the moulds and the replicated structures were investigated by white light interference microscopy (WLI, Micromap 512) and optical microscopy (OM, Jenaval). Additional imaging with scanning electron microscopy (SEM, CARL ZEISS - Ultra 55) and atomic force microscopy (AFM, Dimension FastScan (Bruker), FastScan A cantilever, nominal tip radius $<5 \mathrm{~nm}$ ) were performed. The AFM images were analysed by SPIP software. Copper foils with a thickness of $6 \mu \mathrm{m}$ are used as workpieces. The surface roughness of these foils measured by AFM was about $300 \mathrm{~nm} \mathrm{rms}\left(50 \times 50 \mu \mathrm{m}^{2}\right)$.

\section{Results and discussion}

The mechanical energy which is necessary for the laser embossing (LE) process is provided by the laser ablation of the PI foil and the subsequently generated plasma. The pressure generated by the plasma is transferred to the workpiece through shock waves. Detailed studies of the involved processes at laser ablation are summarized in [2]. The pressure generated by the laser ablation of an area of $200 \times 500 \mu \mathrm{m}^{2}$ has been directly measured by a piezoelectric sensor based on a PVDF and has been calculated from shock wave expansion velocities. As shown in Figure 1 (bottom) the laser-induced pressure increases with the laser 
fluence from $\sim 100 \mathrm{MPa}$ at a laser fluence of $3 \mathrm{~J} / \mathrm{cm}^{2}$ up to a value of $150 \mathrm{MPa}$ at a laser fluence of $5 \mathrm{~J} / \mathrm{cm}^{2}$. The pressure values fit very well with the theoretically predicted pressure values calculated by the model of Phipps [19].

\subsection{Replication of sinusoidal submicron gratings}

Thin nickel foils featuring a two-dimensional, sinusoidal grating with a period of $\sim 0.9 \mu \mathrm{m}$ and a peak-to-valley value $(\mathrm{P}-\mathrm{V})$ of $\sim 400 \mathrm{~nm}$ are applied as master. For the laser embossing into copper the laser beam was scanned meandering ( $\mathrm{v}=2 \mathrm{~mm} / \mathrm{s})$ across the sample with a laser fluence of $F=3.3 \mathrm{~J} / \mathrm{cm}^{2}$. Figure 3 shows AFM images of the master foil (a) and the replicated copper surfaces (b).
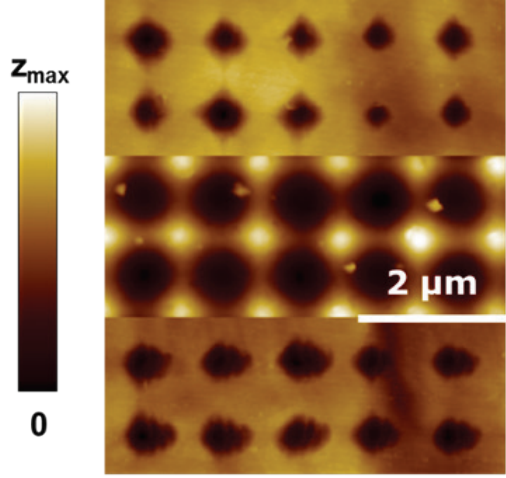

R b)

M a)

$\mathrm{R}$

\section{b)}

Figure 3: AFM images of the master (a) and the replicated (b) sinusoidal submicron gratings $\left(F=3.3 \mathrm{~J} / \mathrm{cm}^{2}\right)$. The feature height of the laser-embossed 2D grating is reduced from $\sim 400 \mathrm{~nm}$ to $\sim$

$230 \mathrm{~nm}$. Further the shape of the replicated patterns changes from rotationally symmetric to square pyramidal. The lower replica image shows shadow-like patterns probably resulting from multiple embossing. The z-scales are $550 \mathrm{~nm}$ for a) and $300 \mathrm{~nm}$ for $\mathrm{b})$.

The embossed copper surface features periodical patterns with the same period but different $\mathrm{P}-\mathrm{V}$ value of $230 \mathrm{~nm}$ (master $\sim 400 \mathrm{~nm}$ ). Obviously not the whole height of the master structures is embossed as can be concluded from the reduced peak-to-valley value and the flat surface between the embossed indentations. The shape of the patterns is rather rectangular than round as expected. The shape and dimension vary within the AFM-imaged surface; the top and the bottom images of the replicated structures show significant differences. Clear squares as well as rhombic shapes can be found. The rhombic-shaped patterns have the same orientation. A detailed analysis suggests that the shape originates from multiple forming processes with a slightly displacement of the two surfaces between the subsequent laser pulses. The different depth of the embossed patterns correlates in parts with the waviness of the surface; a higher average surface results in deeper embossing.

Further, after sectioning the copper sample near the embossed surface by FIB the cross section was imaged by SEM (see Figure 4). The topography of the copper surface features similar characteristics as has been observed by AFM and SEM. Defects (dark spots) and cracks that occur near the surface do not originate from defects of the master but are remaining features from the technical rolling process of the copper foil that causes a surface roughness exceeding $300 \mathrm{~nm}$ rms [20]. Also in this case pattern transfer by laser microembossing is accompanied by some kind of smoothing out the roughness.

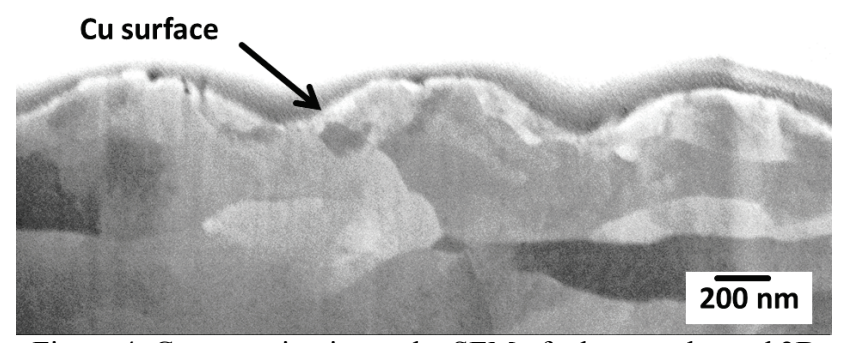

Figure 4: Cross section image by SEM of a laser-embossed 2D grating in copper. For FIB preparation the $\mathrm{Cu}$ surface was protected by a Pt film. The SEM image shows similar topography features as shown by AFM and SEM top view. Near-surface defects and cracks are visible originating from the rolling process. In addition the polycrystalline structure of the copper foil is imaged. Near the surface smaller grains are more frequently visible, however, a clear assignment to the laser embossing process is currently not possible.

\subsection{Replication of LSFL patterns}

Silicon samples featuring LIPSS patterns arranged in lines were replicated into technical copper foils. Optical and SEM images of the embossed LIPSS patterns into a copper surface are seen in different magnifications in Figure 5. As already shown [21] a significant smoothing effect of the micro-roughness occurs within the laser-processed area. However, some waviness, small clefts, e.g. from grain boundaries, and surface irregularities are left that may interfere the embossing of the submicron LIPSS as seen in Figure $5 \mathrm{~b}$ ) and c). The smoothing of the rolled surface is related to the embossing of the flat surface of the silicon wafer surface.
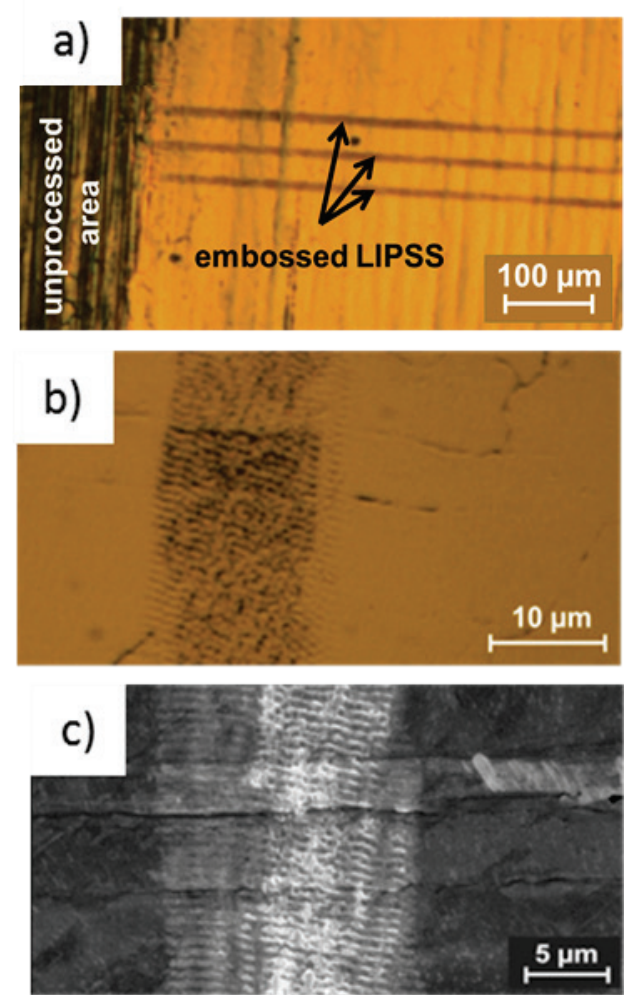

Figure 5: Images of replicated LIPSS patterns into copper foils. a) The optical microscopic image shows the reduction of the surface roughness with the replicated LIPSS-lines at the laserembossed area, b) enlarged optical microimage, and c) SEM image of the LIPSS patterns into a $\mathrm{Cu}$ surface. 
Due to the different height of the samples also after smoothing out the rolling marks the laser-induced pressure is different and accordingly the quality of the replicated structures varies as seen in Figure $5 \mathrm{~b}$ ) and c). This is similar for cracks that have a size similar the ripples period. Already with high optical magnification some further features can be estimated, higher magnifying SEM images clearly show that in addition to the regular LIPSS some scratches appear that do not arise in the master surface. These scratch-like patterns vary in density and length between the samples. A detailed study shows, however, that the scratches are related to the silicon spikes originating from surface-adherent silicon microspheres.
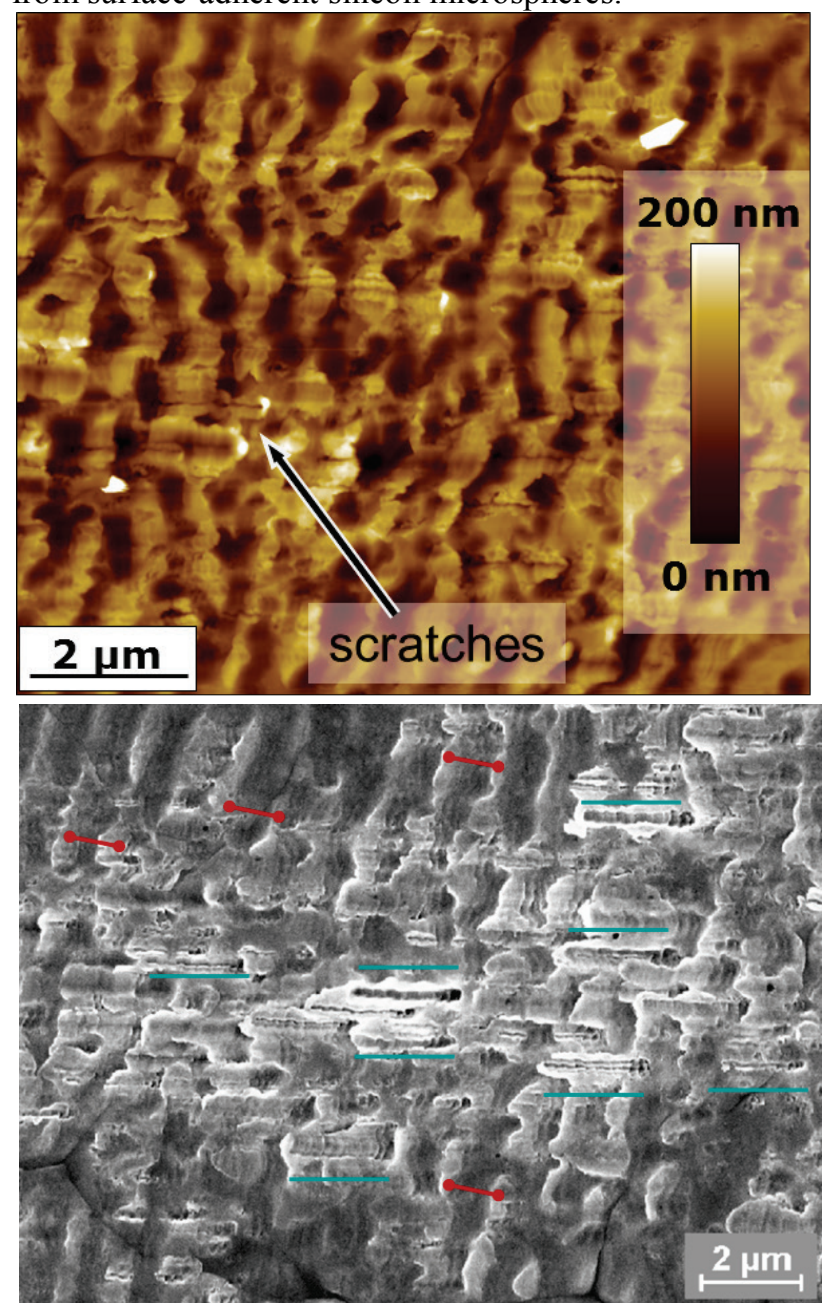

Figure 6: Images of laser-embossed, replicated copper surfaces with the Si-LIPSS: (top) AFM image showing the ripples and scratches and (bottom) SEM image with labels indicating the LIPSS period (red) and the scratches (bluegreen), respectively.

The typical laser-induced ripple pattern but also additional scratch-like features perpendicular to the LIPSS are seen. Compared to Figure 2 showing the silicon master the height of the ripples is reduced and unforeseen patterns appear.

Figure 6 shows an AFM (top) and SEM (bottom) image of laser-embossed LIPSS into a copper foil. In comparison to Figure 2 the ripples are less pronounced. The ripple periods of approx. $750 \mathrm{~nm}$ that are marked in Figure 6 (bottom) by dot-limited lines are much shorter than the scratch-like patterns that are approx. $\sim 2 \mu \mathrm{m}$ long and marked in the image by solid lines and directed in a different direction than the LIPSS. Further, all scratch-like patterns have the same direction and length; in consequence the reason for the formation of these patterns should be the same. As also seen the scratches feature some substructures. The AFM image in (top) also shows that a rim of material is attached to the end of the scratches.

The deep scratches may originate from the high spikes of the master surfaces originating from the coalescence silicon microspheres. The density, the size, and the distribution vary along the LIPSS patterns. The scratches are probably produced during the multi-pulse replication process at which the microspheres are the highest parts of the master and can leave these scratches in the case if the two surfaces move slightly from pulse to pulse. The substructure of the scratches is a sign for this mechanism. Further, at the end of the scratches are rims of copper. During the multi-pulse embossing the copper foils are flattened and pressed so that a slight motion of the copper foil surface relative to the fixed silicon master can be expected. This mechanism might be supported by the fact that between the copper and the silicon surfaces a small gap exists due to the rolling marks, the spikes of the silicon master, and the topography of the laser-processed master.

Further, it has been found, that the length of the scratches depends on the laser spot scanning regime in relation to the laser-written LIPSS lines. Keeping in mind that for scratch generation due to spikes of the master both the replication as well as the relative movement of both surfaces is required it can be explained that in the case of parallel scanning with the laser longer scratches appear because the foil is stretched due to flattening and embossed within the whole spot size whereas at perpendicular scanning only a section of the spot size is modified.

The AFM measurements of the master and corresponding laser-embossed replica have been evaluated by Fourier analyses. The main features - the grating and the ripples period - are dominating the spectra of the masters as well as of the embossed replicas. The evaluation of the sinusoidal master features almost exclusively the spatial frequency of the grating. However, after replication further spatial frequencies both in the low as well as in the high frequency range occur. The low spatial frequencies are remaining rolling defects and the high frequency peaks are harmonics of the grating period and originate from non-sinusoidal rather binary features of the embossed patterns.
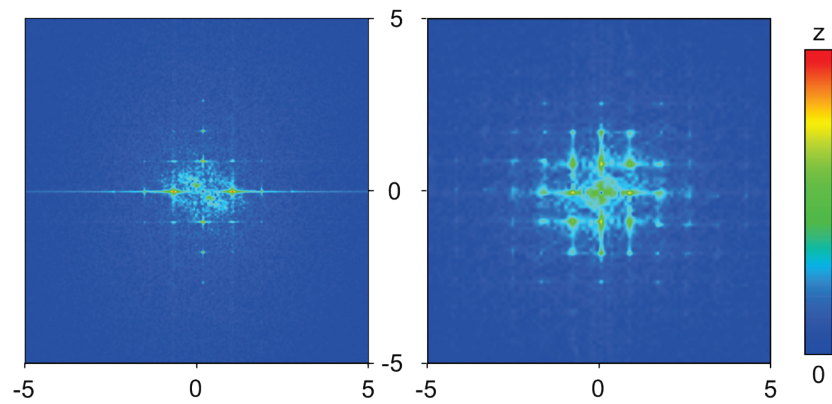

Figure 7: Comparison of the 2D FFT images of the whole AFM images of the master (left) and the replica (right) of Figure 3. The scales are $\mu \mathrm{m}-1$ and $4 \sqrt{ } \mathrm{nm}$ for the lateral and the vertical dimensions, respectively. The master almost exclusively features the spatial frequency of the sinusoidal grating. In contrast, both a higher amount of low spatial frequencies as well as additional peaks - probably a higher order of the grating period - can be found at the replica surface. 
The Fourier analysis of the ripple samples is shown in Figure 8. The basic frequency of $755 \mathrm{~nm}$ that is the main ripples period of the sample can be observed in the master as well as in the samples. Two differences occur: (i) the first overtone (labelled with $373 \mathrm{~nm}$ ) of the spatial frequency of $755 \mathrm{~nm}$ that is clearly visible in the master diminishes in the replicated surfaces and (ii) the intensity of low spatial frequencies probably increases due to the rolling marks from the utilized copper foil.

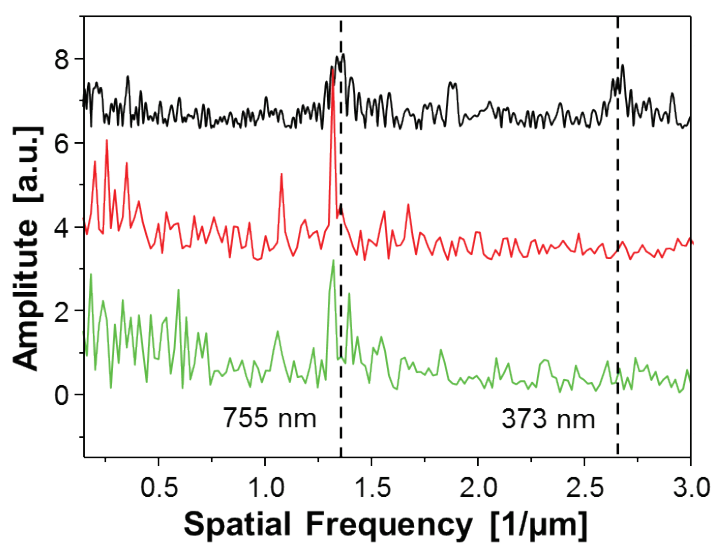

Figure 8: Fourier-transformed AFM cross sections of the master (black) and two replicated surfaces (red/green). The laser scanning direction relative to the LIPSS of the master for the replication process was perpendicularly (green) and horizontally (red), receptivity. The $755 \mathrm{~nm}$ peak is the period of the LIPSS.

The missing $373 \mathrm{~nm}$ peak of the master that represents nonsinusoidal (binary like) topography features of the ripples can be discussed as alteration of the topography of the replicated structures. This is not surprising because laser embossing bases on elastoplastic processes for forming the surface topography by the master. The elastic part of the material formed during embossing relaxes after the embossing. The actual elastic deformation is, however, determined by the topography of the master. Convex edges are normally less good replicated than concave ones as known from other replication technologies in micron technology. This behaviour of the laser embossing process of suppressing of high spatially frequency surface topography features can be discussed controversial. Because replication processes normally aim to achieve identical inverse copies of the master surface such changes of the surface topography are not intended and can be rated to be unfavourable. However, high frequency surface features may be the roughness of surfaces and the filtering of such a roughness within the process might be valuable for some applications. Especially optical applications require a low surface roughness and sometimes continuous profiles like sinusoidal ones.

\section{Conclusion}

Submicron topographical features can be replicated by laser embossing in "normal" configuration meaning the sample foil is on top of the master. The scanning approach of laser embossing allows at one hand a multi-pulse embossing of large areas that is not limited by the laser pulse energy but requires on the other hand a careful implementation of the processing. Especially the displacement of the two surfaces within the multi-pulse embossing has to be prevented to avoid the formation of shadow features. This movement can be originated from insufficient fixing of master or sample or from expansion of one of both. Further, some limitations of the fidelity of the pattern replication process have been observed that can be summarized by topography changes. Further experiments as well as simulations of the laser embossing process should be performed to understand the changes of the topography features and to optimize the whole laser embossing process.

\section{Acknowledgments}

The authors are grateful to Mrs Salamatin for careful correction of the manuscript and to Mrs Mill for support with the SEM and FIB analysis. The partial support of the DAAD under contract 56266271 is acknowledged.

\section{References}

[1] V. Saile et al., LIGA and its Applications, Wiley-VCH Verlag (2008).

[2] D. Bäuerle, Laser Processing and Chemistry, Springer, Berlin, Heidelberg, New York (2011).

[3] G. Raciukaitis et al., J. Laser Micro Nanoeng. 4(2009)186.

[4] D. J. Lipomi et al., 7.11 - Soft Lithographic Approaches to Nanofabrication, In: Polymer Science: A Comprehensive Reference, Ed. K. Matyjaszewski and M. Möller, Elsevier, Amsterdam (2012).

[5] B. Cui et al., Nanotechnology 21(2011)045303.

[6] K. Ding et al., Laser shock peening: Performance and process simulation, CRC Press, (2006).

[7] J. L. Ocana et al., Appl. Surf. Sci. 254(2007)997.

[8] H. Wielage et al., J. Mater. Process. Technol. 211(2011)953.

[9] L. Ocana et al., Appl. Surf. Sci. 255(2009)5633.

[10]H. X. Liu et al., Appl. Surf. Sci. 256(2010)4687.

[11]M. Zhou et al., Appl. Phys. A 90(2008)293.

[12] G. J. Cheng et al., J. Appl. Phys. 101(2007)7.

[13]H. Gao et al., J. Manuf. Sci. Eng.-Trans. ASME 131(2009)11.

[14] J. Li et al., J. Appl. Phys. 108(2010)013107.

[15]Z. B. Shen et al., Mater. Manuf. Process. 26(2011)1126.

[16]H. Wielage et al., Steel Res. Int. 80(2009)323.

[17]H. Wielage et al., J. Mater. Process. Technol. 212(2012)685.

[18] M. Ehrhardt et al., Physics Procedia 39(2012)735.

[19] C. R. Phipps et al., J. Appl. Phys. 64(1988)1083.

[20]M. Ehrhardt et al., J. Laser Micro/Nanoeng. 8(2013)85.

[21]M. Ehrhardt et al., Laser Eng 27(2014)1.

(Received: May 24, 2014, Accepted: October 30, 2014) 